\title{
Parameter independent model order reduction
}

\author{
Lihong Feng ${ }^{a, b, *}$ \\ ${ }^{a}$ ASIC \& System State Key Laboratory, Microelectronics Department, Fudan University, HanDan Road 220, \\ Shanghai 200433, China \\ b IMTEK-Institute for Microsystem Technology, University of Freiburg, D-79110 Freiburg, Germany
}

Received 21 June 2004; received in revised form 2 December 2004; accepted 16 December 2004

Available online 22 January 2005

\begin{abstract}
Several recently developed model order reduction methods for fast simulation of large-scale dynamical systems with two or more parameters are reviewed. Besides, an alternative approach for linear parameter system model reduction as well as a more efficient method for nonlinear parameter system model reduction are proposed in this paper. Comparison between different methods from theoretical elegancy to complexity of implementation are given. By these methods, a large dimensional system with parameters can be reduced to a smaller dimensional parameter system that can approximate the original large sized system to a certain degree for all the parameters.
\end{abstract}

(C) 2004 IMACS. Published by Elsevier B.V. All rights reserved.

Keywords: Model order reduction; Parameter system; Projection technique; Krylov subspace; Arnoldi Process

\section{Introduction}

In recent years, model order reduction techniques (see [1-9] and their references) proved to be promising for fast simulation of large-scale dynamical systems. Almost all the previous work are done on system with one parameter $s$ as follows:

$$
\begin{aligned}
& (s \mathbf{E}+\mathbf{G}) \mathbf{x}(s)=\mathbf{b} u(s) \\
& y(s)=\mathbf{c}^{\mathrm{T}} \mathbf{x}(s)
\end{aligned}
$$

\footnotetext{
* Tel.: +86 216564 2764; fax: +86 2165640850 .

E-mail address: lhfeng@fudan.edu.cn (L. Feng).
} 
where $s$ is a frequency domain parameter, which usually comes from Laplace transform of a linear time domain system below

$$
\begin{aligned}
& \mathbf{E} \frac{\mathrm{d} \mathbf{x}(t)}{\mathrm{d} t}+\mathbf{G} \mathbf{x}(t)=\mathbf{b} u(t) \\
& y(t)=\mathbf{c}^{\mathrm{T}} \mathbf{x}(t)
\end{aligned}
$$

There are many engineering problems that a model like (3) with more parameters are needed to give a more accurate description of the problem. Therefore, some more general model order reduction techniques [1013] are newly developed to deal with this kind of model. These methods are basically generalization of the traditional model order reduction methods for one parameter systems based on projection techniques $[3,5,6]$,

$$
\begin{aligned}
& \left(\mathbf{E}_{0}+s_{1} \mathbf{E}_{1}+s_{2} \mathbf{E}_{2}+\cdots+s_{p} \mathbf{E}_{p}\right) \mathbf{x}\left(s_{1}, s_{2}, \ldots, s_{p}\right)=\mathbf{b} u\left(s_{1}, s_{2}, \ldots, s_{p}\right) \\
& y\left(s_{1}, s_{2}, \ldots, s_{p}\right)=\mathbf{c}^{\mathrm{T}} \mathbf{x}\left(s_{1}, s_{2}, \ldots, s_{p}\right)
\end{aligned}
$$

where $\mathbf{E}_{0}, \mathbf{E}_{1}, \ldots, \mathbf{E}_{p} \in R^{n \times n}$ are system matrices. $\mathbf{b} \in R^{n \times l}, \mathbf{c} \in R^{n \times m}$ are matrices that relate the input $u$ to the output $y . u$ is a known function, $\mathbf{x} \in R^{n}$ is the unknown vector. Here for simplicity, we only consider system with $l=1, m=1$, that is, $\mathbf{b}$ and $\mathbf{c}$ are all vectors.

The purpose of parameter independent order reduction is to find a much smaller dimensional system

$$
\begin{aligned}
& \left(\hat{\mathbf{E}}_{0}+s_{1} \hat{\mathbf{E}}_{1}+s_{2} \hat{\mathbf{E}}_{2}+\cdots+s_{p} \hat{\mathbf{E}}_{p}\right) \mathbf{z}\left(s_{1}, s_{2}, \ldots, s_{p}\right)=\hat{\mathbf{b}} u\left(s_{1}, s_{2}, \ldots, s_{p}\right) \\
& \hat{y}\left(s_{1}, s_{2}, \ldots, s_{p}\right)=\hat{\mathbf{c}}^{\mathrm{T}} \mathbf{z}\left(s_{1}, s_{2}, \ldots, s_{p}\right)
\end{aligned}
$$

with $\mathbf{z} \in R^{q}, q \ll n$; moreover, the error between $\hat{y}$ and $y$ can be acceptable for all parameters $s_{1}, s_{2}, \ldots, s_{p}$ in certain engineering applications, i.e. $\|y-\hat{y}\|<\varepsilon, \forall s_{i}, i=1,2, \ldots, p$ for a given a tolerance $\varepsilon$. In the end, the simulation of the original large system (3) can be replaced by simulation of the reduced smaller system (4), which can largely speed up the simulation process.

In the following text, we will first give a short introduction of the general idea of traditional model reduction methods with projection technique in Section 2. We analyze different parameter order reduction methods on system (3) in Section 3. Besides, we propose an alternative parameter independent order reduction method. In Section 4, we discuss model reduction on parameter system which is nonlinearly dependent on the parameters and show an improved approach based on the known methods. Finally, some conclusions are given in Section 5.

\section{Traditional one parameter model order reduction}

Traditional projection model order reduction method can only deal with system (3) with one parameter, it is to find projection matrices $\mathbf{W} \in R^{n \times q}, \mathbf{V} \in R^{n \times q}, q \ll n$ satisfying $\mathbf{W}^{\mathrm{T}} \mathbf{V}=\mathbf{I}$ such that the reduced linear system is

$$
\begin{aligned}
& \left(\hat{\mathbf{E}}_{0}+s \hat{\mathbf{E}}_{1}\right) \mathbf{z}(s)=\hat{\mathbf{b}} u(s) \\
& \hat{y}(s)=\hat{\mathbf{c}}^{\mathrm{T}} \mathbf{z}(s)
\end{aligned}
$$

where $\hat{\mathbf{E}}_{0}=\mathbf{W}^{\mathrm{T}} \mathbf{E}_{0} \mathbf{V}, \hat{\mathbf{E}}_{1}=\mathbf{W}^{\mathrm{T}} \mathbf{E}_{1} \mathbf{V}, \hat{\mathbf{b}}=\mathbf{W}^{\mathrm{T}} \mathbf{b}, \hat{\mathbf{c}}^{\mathrm{T}}=\mathbf{c}^{\mathrm{T}} \mathbf{V}$. I is the identity matrix. The projection matrices $\mathbf{W}, \mathbf{V}$ can be identical [3] or different [2]. In the following, we assume they are identical. 
According to the transfer function

$$
h(s)=\mathbf{c}^{\mathrm{T}}\left(\mathbf{E}_{0}+\mathbf{E}_{1} s\right)^{-1} \mathbf{b}=\mathbf{c}^{\mathrm{T}}\left(\mathbf{I}-\left(-\mathbf{E}_{0}^{-1} \mathbf{E}_{1}\right) s\right)^{-1} \mathbf{E}_{0}^{-1} \mathbf{b}=\mathbf{c}^{\mathrm{T}} \sum_{i=0}^{\infty}\left(-\mathbf{E}_{0}^{-1} \mathbf{E}_{1}\right)^{i} \mathbf{E}_{0}^{-1} \mathbf{b} s^{i}
$$

The projection matrix is constructed as orthogonal bases of the subspace below

$$
\operatorname{spancol}\{\mathbf{V}\}=\operatorname{spancol}\left\{\mathbf{E}_{0}^{-1} \mathbf{b},\left(-\mathbf{E}_{0}^{-1} \mathbf{E}\right) \mathbf{E}_{0}^{-1} \mathbf{b},\left(-\mathbf{E}_{0}^{-1} \mathbf{E}\right)^{2} \mathbf{E}_{0}^{-1} \mathbf{b}, \ldots,\left(-\mathbf{E}_{0}^{-1} \mathbf{E}\right)^{J} \mathbf{E}_{0}^{-1} \mathbf{b}\right\}
$$

The right-hand side is just a Krylov subspace defined as,

$$
K_{J+1}\left\{-\mathbf{E}_{0}^{-1} \mathbf{E}, \mathbf{E}_{0}^{-1} \mathbf{b}\right\}
$$

therefore, the Arnoldi Process [6] can be employed to implicitly generate $\mathbf{V}$ without causing numerical instability [2]. In this way, the transfer function of the original system (6) can be approximated by the reduced model: the derivatives (moments) of the transfer function that are included in the Krylov subspace are identical to those of the transfer function of the reduced linear system (5). The more moments are included in (7), the more accurate the reduced model $[5,6]$.

\section{Parameter independent model order reduction}

To be clearly expounded, we first show the basic ideas of different parameter independent model order reduction methods with a two parameter system, that is, in (3) $p=2$. Then, we generalize them to any $p$ parameters. The goal of all the methods below is to find an orthogonal projection matrix $\mathbf{V}, \mathbf{V}^{\mathrm{T}} \mathbf{V}=\mathbf{I}$, such that the reduced system is derived by the manipulation of the projection below:

$$
\begin{aligned}
& \left(\hat{\mathbf{E}}_{0}+s_{1} \hat{\mathbf{E}}_{1}+s_{2} \hat{\mathbf{E}}_{2}\right) \mathbf{z}\left(s_{1}, s_{2}\right)=\hat{\mathbf{b}} u\left(s_{1}, s_{2}\right) \\
& \hat{y}\left(s_{1}, s_{2}\right)=\hat{\mathbf{c}}^{\mathrm{T}} \mathbf{z}\left(s_{1}, s_{2}\right) \\
\hat{\mathbf{E}}_{j}= & \mathbf{V}^{\mathrm{T}} \mathbf{E}_{j} \mathbf{V}, \quad j=0,1,2, \quad \hat{\mathbf{b}}=\mathbf{V}^{\mathrm{T}} \mathbf{b}, \quad \hat{\mathbf{c}}^{\mathrm{T}}=\mathbf{c}^{\mathrm{T}} \mathbf{V} .
\end{aligned}
$$

\subsection{Method 1}

The earlier proposed method is [10]. The transfer function with parameters is defined as

$$
h\left(s_{1}, s_{2}\right)=\mathbf{c}^{\mathrm{T}}\left(\mathbf{E}_{0}+s_{1} \mathbf{E}_{1}+s_{2} \mathbf{E}_{2}\right)^{-1} \mathbf{b}
$$

Similar with one parameter model reduction method, Taylor expansion of $h\left(s_{1}, s_{2}\right)$ is first calculated

$$
h\left(s_{1}, s_{2}\right)=\mathbf{c}^{\mathrm{T}}\left\{\mathbf{I}-\left[-\left(s_{1} \mathbf{E}_{0}^{-1} \mathbf{E}_{1}+s_{2} \mathbf{E}_{0}^{-1} \mathbf{E}_{2}\right)\right]\right\}^{-1} \mathbf{E}_{0}^{-1} \mathbf{b}=\mathbf{c}^{\mathrm{T}} \sum_{i=0}^{\infty}\left[-\left(s_{1} \mathbf{E}_{0}^{-1} \mathbf{E}_{1}+s_{2} \mathbf{E}_{0}^{-1} \mathbf{E}_{2}\right)\right]^{i} \mathbf{E}_{0}^{-1} \mathbf{b}
$$


each term in the series above is further expanded,

$$
h\left(s_{1}, s_{2}\right)=\mathbf{c}^{\mathrm{T}} \sum_{i=0}^{\infty} \sum_{j=0}^{i}\left[\mathbf{F}_{j}^{i}\left(\mathbf{E}_{0}^{-1} \mathbf{E}_{1}, \mathbf{E}_{0}^{-1} \mathbf{E}_{2}\right) s_{1}^{i-j} s_{2}^{j}\right] \mathbf{E}_{0}^{-1} \mathbf{b}
$$

where $\mathbf{F}_{j}^{i}$ are the multipliers before $s_{1}^{i-j} s_{2}^{j}$, when $\left[-\left(s_{1} \mathbf{E}_{0}^{-1} \mathbf{E}_{1}+s_{2} \mathbf{E}_{0}^{-1} \mathbf{E}_{2}\right)\right]^{i}$ are expanded into separate terms. For example, when $i=2,\left[-\left(s_{1} \mathbf{E}_{0}^{-1} \mathbf{E}_{1}+s_{2} \mathbf{E}_{0}^{-1} \mathbf{E}_{2}\right)\right]^{2}$ is expanded into three terms,

$$
\left(E_{0}^{-1} E_{1}\right)^{2} s_{1}^{2},\left[\left(E_{0}^{-1} E_{1}\right)\left(E_{0}^{-1} E_{2}\right)+\left(E_{0}^{-1} E_{2}\right)\left(E_{0}^{-1} E_{1}\right)\right] s_{1} s_{2},\left(E_{0}^{-1} E_{2}\right)^{2} s_{2}^{2}
$$

each term corresponds to $F_{0}^{2}, F_{1}^{2}, F_{2}^{2}$, respectively, that is

$$
\left(E_{0}^{-1} E_{1}\right)^{2}=F_{0}^{2},\left[\left(E_{0}^{-1} E_{1}\right)\left(E_{0}^{-1} E_{2}\right)+\left(E_{0}^{-1} E_{2}\right)\left(E_{0}^{-1} E_{1}\right)\right] s_{1} s_{2}=F_{1}^{2},\left(E_{0}^{-1} E_{2}\right)^{2}=F_{2}^{2}
$$

The projection matrix $\mathbf{V}$ is constructed based on the terms $\mathbf{F}_{j}^{i}\left(\mathbf{E}_{0}^{-1} \mathbf{E}_{1}, \mathbf{E}_{0}^{-1} \mathbf{E}_{2}\right) \mathbf{E}_{0}^{-1} \mathbf{b}$ in (10),

$$
\operatorname{spancol}\{\mathbf{V}\}=\operatorname{spancol}\left\{\bigcup_{m=0}^{J}\left(\bigcup_{k=0}^{m} \mathbf{F}_{k}^{m}\left(\mathbf{E}_{0}^{-1} \mathbf{E}_{1}, \mathbf{E}_{0}^{-1} \mathbf{E}_{2}\right) \mathbf{E}_{0}^{-1} \mathbf{b}\right)\right\}
$$

It has been proved in [10] that by this projection matrix, the reduced system can match the terms $\mathbf{F}_{j}^{i}$ that included in the right-hand side of (12), that is

$$
\mathbf{c}^{\mathrm{T}} \mathbf{F}_{k}^{m}\left(\mathbf{E}_{0}^{-1} \mathbf{E}_{1}, \mathbf{E}_{0}^{-1} \mathbf{E}_{2}\right) \mathbf{E}_{0}^{-1} \mathbf{b}=\hat{\mathbf{c}}^{\mathrm{T}} \mathbf{F}_{k}^{m}\left(\hat{\mathbf{E}}_{0}^{-1} \hat{\mathbf{E}}_{1}, \hat{\mathbf{E}}_{0}^{-1} \hat{\mathbf{E}}_{2}\right) \hat{\mathbf{E}}_{0}^{-1} \hat{\mathbf{b}}, \quad k=0, \ldots, m, \quad m=0, \ldots, J
$$

\subsection{Method 2}

Another method is proposed in [12]. This method is also based on projection technique (8) with the projection matrix $\mathbf{V}$ being computed in a different way.

By this method, the derivatives of $\mathbf{x}\left(s_{1}, s_{2}\right)$ w.r.t. $s_{1}, s_{2}$ are computed separately. The derivatives of one parameter is estimated when the other parameter is fixed as a constant number, for example, derivatives w.r.t. $s_{1}$ at $s_{1}=0$ is computed by fixing $s_{2}=s_{2}^{0}$; here, we let $s_{2}^{0}=0$ and denote $M_{i}^{s_{1}}$ the $i$ th order derivative of $\mathbf{x}$ w.r.t. $s_{1}$, then from (3)

$$
\left(\mathbf{E}_{0}+s_{1} \mathbf{E}_{1}\right)\left[M_{0}^{s_{1}}+M_{1}^{s_{1}} s_{1}+\cdots \frac{M_{j}^{s_{1}}}{j !}\left(s_{1}\right)^{j}+\cdots\right]=\mathbf{b} u
$$

Like powers of $s_{1}$ being equated, we have

$$
\begin{aligned}
& \mathbf{E}_{0} M_{0}^{s_{1}}=\mathbf{b} u \\
& \mathbf{E}_{0} M_{j}^{s_{1}}=-j \mathbf{E}_{1} M_{j-1}^{s_{1}}, \quad j=1,2, \ldots
\end{aligned}
$$

Derivatives $M_{i}^{s_{2}}, i=0,1, \ldots$ w.r.t. $s_{2}$ at $s_{2}=0$ can also be computed in the same way. Note, here $M_{j}^{s_{1}}$ is slightly different from those in [12], where $M_{j}^{s_{1}}$ is defined as moments of $\mathbf{x}$, and the coefficients $\frac{1}{j !}$ is just included into $M_{j}^{s_{1}}$. In the end, the projection matrix $\mathbf{V}$ is constructed as

$$
\operatorname{spancol}\{\mathbf{V}\}=\operatorname{spancol}\left\{M_{j}^{s_{1}}, M_{i}^{s_{2}}, j=0,1, \ldots, J, i=0,1, \ldots, I\right\}
$$


The theorem offered in [12] confirms that the derivatives included in $\mathbf{V}$ can be conserved by the reduced system, that is

$$
M_{j}^{s_{1}}=\mathbf{V} \hat{M}_{j}^{s_{1}}, \quad j=0,1, \ldots, J ; \quad M_{i}^{s_{2}}=\mathbf{V} \hat{M}_{i}^{s_{2}}, \quad i=0,1, \ldots, I
$$

where $\hat{M}_{j}^{s_{1}}, \hat{M}_{j}^{s_{2}}$ is the derivatives of $\mathbf{z}$ in the reduced model (8). Then we have that the corresponding derivatives of $y$ and $\hat{y}$ are identical, i.e. $\hat{\mathbf{c}}^{\mathrm{T}} \hat{M}_{j}^{s_{i}}=\mathbf{c}^{\mathrm{T}} \mathbf{V} \mathbf{V}^{\mathrm{T}} \boldsymbol{M}_{j}^{s_{i}}=\mathbf{c}^{\mathrm{T}} M_{j}^{s_{i}}, i=1,2$. The last identity $\mathbf{c}^{\mathrm{T}} \mathbf{V} \mathbf{V}^{\mathrm{T}} \boldsymbol{M}_{j}^{s_{i}}=\mathbf{c}^{\mathrm{T}} M_{j}^{s_{i}}$ can be proved by Lemma 3.3 in Section 3.3.

\subsection{Method 3}

In this section, we give another way of constructing $\mathbf{V}$. This can be done by series expansion of $h\left(s_{1}, s_{2}\right)$ w.r.t. the parameters one after another, that is, we first expand $h\left(s_{1}, s_{2}\right)$ w.r.t. $s_{1}$ into Taylor series at expansion point $s_{1}=0$,

$$
\begin{aligned}
h\left(s_{1}, s_{2}\right) & =\mathbf{c}^{\mathrm{T}}\left(\mathbf{E}_{0}+s_{1} \mathbf{E}_{1}+s_{2} \mathbf{E}_{2}\right)^{-1} \mathbf{b}=\mathbf{c}^{\mathrm{T}}\left[\mathbf{I}-\left(-\left(\mathbf{E}_{0}+s_{2} \mathbf{E}_{2}\right)^{-1} s_{1} \mathbf{E}_{1}\right)\right]^{-1}\left(\mathbf{E}_{0}+s_{2} \mathbf{E}_{2}\right)^{-1} \mathbf{b} \\
& =\mathbf{c}^{\mathrm{T}} \sum_{i=0}^{\infty}\left[-\left(\mathbf{E}_{0}+s_{2} \mathbf{E}_{2}\right)^{-1} \mathbf{E}_{1}\right]^{i}\left(\mathbf{E}_{0}+s_{2} \mathbf{E}_{2}\right)^{-1} \mathbf{b} s_{1}^{i}=\mathbf{c}^{\mathrm{T}} \sum_{i=0}^{\infty} M_{i}\left(s_{2}\right) s_{1}^{i}
\end{aligned}
$$

where $M_{i}\left(s_{2}\right)=\left[-\left(\mathbf{E}_{0}+s_{2} \mathbf{E}_{2}\right)^{-1} \mathbf{E}_{1}\right]^{i}\left(\mathbf{E}_{0}+s_{2} \mathbf{E}_{2}\right)^{-1} \mathbf{b}$. Each $M_{i}\left(s_{2}\right)$ can further be expanded into series of $s_{2}$,

$$
\begin{aligned}
M_{i}\left(s_{2}\right)= & {\left[-\left(\mathbf{E}_{0}+s_{2} \mathbf{E}_{2}\right)^{-1} \mathbf{E}_{1}\right]^{i}\left(\mathbf{E}_{0}+s_{2} \mathbf{E}_{2}\right)^{-1} \mathbf{b}=-\left(\mathbf{E}_{0}+s_{2} \mathbf{E}_{2}\right)^{-1} \mathbf{E}_{1} M_{i-1}\left(s_{2}\right) } \\
= & -\left(\mathbf{I}-\left(-s_{2} \mathbf{E}_{0}^{-1} \mathbf{E}_{2}\right)\right)^{-1} \mathbf{E}_{0}^{-1} \mathbf{E}_{1} M_{i-1}\left(s_{2}\right)=-\sum_{j_{i}=0}^{\infty}\left(-\mathbf{E}_{0}^{-1} \mathbf{E}_{2}\right)^{j_{i}} \mathbf{E}_{0}^{-1} \mathbf{E}_{1} s_{2}^{j_{i}} M_{i-1}\left(s_{2}\right) \\
= & \left\{-\sum_{j_{i}=0}^{\infty}\left(-\mathbf{E}_{0}^{-1} \mathbf{E}_{2}\right)^{j_{i}} \mathbf{E}_{0}^{-1} \mathbf{E}_{1}\right\}\left\{-\sum_{j_{i-1}=0}^{\infty}\left(-\mathbf{E}_{0}^{-1} \mathbf{E}_{2}\right)^{j_{i-1}} \mathbf{E}_{0}^{-1} \mathbf{E}_{1}\right\} s_{2}^{j_{i}} s_{2}^{j_{i-1}} M_{i-2}\left(s_{2}\right)=\vdots \\
= & (-1)^{i} \prod_{t=1}^{i}\left(\sum_{j_{t}=0}^{\infty}\left(-\mathbf{E}_{0}^{-1} \mathbf{E}_{2}\right)^{j_{t}} \mathbf{E}_{0}^{-1} \mathbf{E}_{1} s_{2}^{j_{t}}\right) M_{0}\left(s_{2}\right) \\
= & (-1)^{i} \prod_{t=1}^{i}\left(\sum_{j_{t}=0}^{\infty}\left(-\mathbf{E}_{0}^{-1} \mathbf{E}_{2}\right)^{j_{t}} \mathbf{E}_{0}^{-1} \mathbf{E}_{1} s_{2}^{j_{t}}\right)\left(\sum_{j_{0}=0}^{\infty}\left(-\mathbf{E}_{0}^{-1} \mathbf{E}_{2}\right)^{j_{0}} \mathbf{E}_{0}^{-1} \mathbf{b}_{2}^{j_{0}}\right) \\
= & \sum_{j_{i}=0}^{\infty} \ldots \sum_{j_{1}=0}^{\infty} \sum_{j_{0}=0}^{\infty}\left(-\mathbf{E}_{0}^{-1} \mathbf{E}_{2}\right)^{j_{i}} \mathbf{E}_{0}^{-1} \mathbf{E}_{1} \ldots\left(-\mathbf{E}_{0}^{-1} \mathbf{E}_{2}\right)^{j_{1}} \mathbf{E}_{0}^{-1} \mathbf{E}_{1}\left(-\mathbf{E}_{0}^{-1} \mathbf{E}_{2}\right)^{j_{0}} \mathbf{E}_{0}^{-1} \mathbf{b} s_{2}^{j_{i}} s_{2}^{j_{i-1}} \ldots, s_{2}^{j_{0}} \\
= & \sum_{j_{i}=0}^{\infty} \ldots \sum_{j_{1}=0}^{\infty} \sum_{j_{0}=0}^{\infty} \mathbf{E}_{\left.j_{i}, j_{i-1}, \ldots, j_{0}\right) s_{2}^{j_{i}} s_{2}^{j_{i-1}} \ldots, s_{2}^{j_{0}}}
\end{aligned}
$$


from (17), we can see that each $M_{i}, i=0,1,2, \ldots$ is just linear combination of $\mathbf{E}\left(j_{i}, j_{i-1}, \ldots, j_{0}\right)$ which is independent of all the parameters. Since $\mathbf{x}\left(s_{1}, s_{2}\right)$ is in the subspace spanned by $M_{i}, i=0,1,2, \ldots$, it is actually in the subspace spanned by $\mathbf{E}\left(j_{i}, j_{i-1}, \ldots, j_{0}\right)$. To this understanding, the projection matrix is constructed as

$$
\begin{aligned}
& \operatorname{spancol}\{\mathbf{V}\}=\operatorname{spancol}\left\{\mathbf{V}_{0}, \mathbf{V}_{1}, \ldots, \mathbf{V}_{I}\right\} \\
& \operatorname{spancol}\left\{\mathbf{V}_{i}\right\}=\operatorname{spancol}\left\{\bigcup_{i=0}^{I} \bigcup_{t=0}^{i} \bigcup_{j_{t}=0}^{q_{t}^{[i]}} \mathbf{E}\left(j_{t}, j_{t-1}, \ldots, j_{0}\right)\right\}
\end{aligned}
$$

Next, we will prove that,

Theorem 3.1. All $\mathbf{E}\left(j_{t}, j_{t-1}, \ldots, j_{0}\right), j_{t}=0,1, \ldots, q_{t}^{[i]}, t=0,1, \ldots, i, i=0,1, \ldots$, I included in the projection matrix $\mathbf{V}$ will be conserved by the reduced model (8) if $\mathbf{V}$ is constructed by (18) and (19), i.e. for $j_{t}=0,1, \ldots, q_{t}^{[i]}, t=0,1, \ldots, i, i=0,1, \ldots, I$

$$
\mathbf{E}\left(j_{t}, j_{t-1}, \ldots, j_{0}\right)=\mathbf{V} \hat{\mathbf{E}}\left(j_{t}, j_{t-1}, \ldots, j_{0}\right) .
$$

Theorem 3.2. All the moments of the original transfer function (16) included in the projection matrix $\mathbf{V}$ in (18) are identical with those of the transfer function of the reduced system (8), i.e. for $j_{t}=0,1, \ldots, q_{t}^{[i]}, t=0,1, \ldots, i, i=0,1, \ldots, I$

$$
c^{\mathrm{T}} \mathbf{E}\left(j_{t}, j_{t-1}, \ldots, j_{0}\right)=\hat{c}^{\mathrm{T}} \hat{\mathbf{E}}\left(j_{t}, j_{t-1}, \ldots, j_{0}\right)
$$

To prove Theorem 3.1, we have to employ a Lemma in [10] without proof,

Lemma 3.3. $\mathbf{V}$ is an orthogonal matrix satisfying $\mathbf{V}^{\mathrm{T}} \mathbf{V}=\mathbf{I}$, then for any $\mathbf{w} \in \operatorname{spancol}\{\mathbf{V}\}, \mathbf{V} \mathbf{V}^{\mathrm{T}} \mathbf{w}=\mathbf{w}$.

Proof of Theorem 3.1. We first prove that for any $j_{0} \leq q_{0}^{[i]}, i=0,1, \ldots, I, \mathbf{E}\left(j_{0}\right)=\mathbf{V} \hat{\mathbf{E}}\left(j_{0}\right)$, i.e.

$$
\left(-\mathbf{E}_{0}^{-1} \mathbf{E}_{2}\right)^{j_{0}} \mathbf{E}_{0}^{-1} \mathbf{b}=\mathbf{V}\left(-\hat{\mathbf{E}}_{0}^{-1} \hat{\mathbf{E}}_{2}\right)^{j_{0}} \hat{\mathbf{E}}_{0}^{-1} \hat{\mathbf{b}}
$$

We start from $j_{0}=0$, since $\mathbf{E}_{0}^{-1} \mathbf{b} \in \operatorname{spancol}\{\mathbf{V}\}$, using Lemma 3.3 we have

$$
\hat{\mathbf{E}}_{0} \mathbf{V}^{\mathrm{T}} \mathbf{E}_{0}^{-1} \mathbf{b}=\mathbf{V}^{\mathrm{T}} \mathbf{E}_{0} \mathbf{V} \mathbf{V}^{\mathrm{T}} \mathbf{E}_{0}^{-1} \mathbf{b}=\mathbf{V}^{\mathrm{T}} \mathbf{E}_{0} \mathbf{E}_{0}^{-1} \mathbf{b}=\mathbf{V}^{\mathrm{T}} \mathbf{b}=\hat{\mathbf{b}}
$$

i.e. $\mathbf{V}^{\mathrm{T}} \mathbf{E}_{0}^{-1} \mathbf{b}=\hat{\mathbf{E}}_{0}^{-1} \hat{\mathbf{b}}$. Again by Lemma $3.3, \mathbf{E}_{0}^{-1} \mathbf{b}=\mathbf{V} \hat{\mathbf{E}}_{0}^{-1} \hat{\mathbf{b}}$.

Suppose that (20) holds for $j_{0}-1$, i.e. $\left(\mathbf{E}_{0}^{-1} \mathbf{E}_{2}\right)^{j_{0}-1} \mathbf{E}_{0}^{-1} \mathbf{b}=\mathbf{V}\left(-\hat{\mathbf{E}}_{0}^{-1} \hat{\mathbf{E}}_{2}\right)^{j_{0}-1} \hat{\mathbf{E}}_{0}^{-1} \hat{\mathbf{b}}$. We next prove that it also holds for $j_{0}$, since $\left(-\mathbf{E}_{0}^{-1} \mathbf{E}_{2}\right)^{j_{0}} \mathbf{E}_{0}^{-1} \mathbf{b} \in \operatorname{spancol}\{\mathbf{V}\}$, by Lemma 3.3

$$
\begin{aligned}
\hat{\mathbf{E}}_{0} \mathbf{V}^{\mathrm{T}}\left(-\mathbf{E}_{0}^{-1} \mathbf{E}_{2}\right)^{j_{0}} \mathbf{E}_{0}^{-1} \mathbf{b} & =\mathbf{V}^{\mathrm{T}} \mathbf{E}_{0} \mathbf{V} \mathbf{V}^{\mathrm{T}}\left(-\mathbf{E}_{0}^{-1} \mathbf{E}_{2}\right)^{j_{0}} \mathbf{E}_{0}^{-1} \mathbf{b}=\mathbf{V}^{\mathrm{T}} \mathbf{E}_{0}\left(-\mathbf{E}_{0}^{-1} \mathbf{E}_{2}\right)^{j_{0}} \mathbf{E}_{0}^{-1} \mathbf{b} \\
& =\mathbf{V}^{\mathrm{T}} \mathbf{E}_{0}\left(-\mathbf{E}_{0}^{-1} \mathbf{E}_{2}\right)\left(-\mathbf{E}_{0}^{-1} \mathbf{E}_{2}\right)^{j_{0}-1} \mathbf{E}_{0}^{-1} \mathbf{b}=-\mathbf{V}^{\mathrm{T}} \mathbf{E}_{2}\left(-\mathbf{E}_{0}^{-1} \mathbf{E}_{2}\right)^{j_{0}-1} \mathbf{E}_{0}^{-1} \mathbf{b} \\
& =-\mathbf{V}^{\mathrm{T}} \mathbf{E}_{2} \mathbf{V}\left(-\hat{\mathbf{E}}_{0}^{-1} \hat{\mathbf{E}}_{2}\right)^{j_{0}-1} \hat{\mathbf{E}}_{0}^{-1} \hat{\mathbf{b}}=-\hat{\mathbf{E}}_{2}\left(-\hat{\mathbf{E}}_{0}^{-1} \hat{\mathbf{E}}_{2}\right)^{j_{0}-1} \hat{\mathbf{E}}_{0}^{-1} \hat{\mathbf{b}},
\end{aligned}
$$

i.e. $\mathbf{V}^{\mathrm{T}}\left(-\mathbf{E}_{0}^{-1} \mathbf{E}_{2}\right)^{j_{0}} \mathbf{E}_{0}^{-1} \mathbf{b}=-\hat{\mathbf{E}}_{0}^{-1} \hat{\mathbf{E}}_{2}\left(-\hat{\mathbf{E}}_{0}^{-1} \hat{\mathbf{E}}_{2}\right)^{j_{0}-1} \hat{\mathbf{E}}_{0}^{-1} \hat{\mathbf{b}}$.

By Lemma 3.3, $\left(-\mathbf{E}_{0}^{-1} \mathbf{E}_{2}\right)^{j_{0}} \mathbf{E}_{0}^{-1} \mathbf{b}=\mathbf{V}\left(-\hat{\mathbf{E}}_{0}^{-1} \hat{\mathbf{E}}_{2}\right)\left(-\hat{\mathbf{E}}_{0}^{-1} \hat{\mathbf{E}}_{2}\right)^{j_{0}-1} \hat{\mathbf{E}}_{0}^{-1} \hat{\mathbf{b}}$, i.e. $\mathbf{E}\left(j_{0}\right)=\mathbf{V} \hat{\mathbf{E}}\left(j_{0}\right)$; thus, we finished the proof with $j_{0} \leq q_{0}^{[i]}, i=0,1, \ldots, I$. 
Now suppose that for any $j_{t} \leq q_{t}^{[i]}, i=0,1, \ldots, I$, Theorem 3.1 holds until for $j_{t}-1$, i.e.

$$
\left(-\mathbf{E}_{0}^{-1} \mathbf{E}_{2}\right)^{j_{t}-1} \mathbf{E}\left(j_{t-1}, j_{t-2}, \ldots, j_{0}\right)=\mathbf{V}\left(-\hat{\mathbf{E}}_{0}^{-1} \hat{\mathbf{E}}_{2}\right)^{j_{t}-1} \hat{\mathbf{E}}\left(j_{t-1}, j_{t-2}, \ldots, j_{0}\right)
$$

We next prove that it also holds for $j_{t}$. Because $\left(-\mathbf{E}_{0}^{-1} \mathbf{E}_{2}\right)^{j_{t}} \mathbf{E}\left(j_{t-1}, j_{t-2}, \ldots, j_{0}\right) \in$ spancol $\{\mathbf{V}\}$, using Lemma 3.3

$$
\begin{aligned}
\hat{\mathbf{E}}_{0} \mathbf{V}^{\mathrm{T}}\left(-\mathbf{E}_{0}^{-1} \mathbf{E}_{2}\right)^{j_{t}} \mathbf{E}\left(j_{t-1}, j_{t-2}, \ldots, j_{0}\right) & =\mathbf{V}^{\mathrm{T}} \mathbf{E}_{0} \mathbf{V} \mathbf{V}^{\mathrm{T}}\left(-\mathbf{E}_{0}^{-1} \mathbf{E}_{2}\right)^{j_{t}} \mathbf{E}\left(j_{t-1}, j_{t-2}, \ldots, j_{0}\right) \\
& =\mathbf{V}^{\mathrm{T}} \mathbf{E}_{0}\left(-\mathbf{E}_{0}^{-1} \mathbf{E}_{2}\right)\left(-\mathbf{E}_{0}^{-1} \mathbf{E}_{2}\right)^{j_{t}-1} \mathbf{E}\left(j_{t-1}, j_{t-2}, \ldots, j_{0}\right) \\
& =-\mathbf{V}^{\mathrm{T}} \mathbf{E}_{2}\left(-\mathbf{E}_{0}^{-1} \mathbf{E}_{2}\right)^{j_{t}-1} \mathbf{E}\left(j_{t-1}, j_{t-2}, \ldots, j_{0}\right) \\
& =-\mathbf{V}^{\mathrm{T}} \mathbf{E}_{2} \mathbf{V}\left(-\hat{\mathbf{E}}_{0}^{-1} \hat{\mathbf{E}}_{2}\right)^{j_{t}-1} \hat{\mathbf{E}}\left(j_{t-1}, j_{t-2}, \ldots, j_{0}\right) \\
& =-\hat{\mathbf{E}}_{2}\left(-\hat{\mathbf{E}}_{0}^{-1} \hat{\mathbf{E}}_{2}\right)^{j_{t}-1} \hat{\mathbf{E}}\left(j_{t-1}, j_{t-2}, \ldots, j_{0}\right)
\end{aligned}
$$

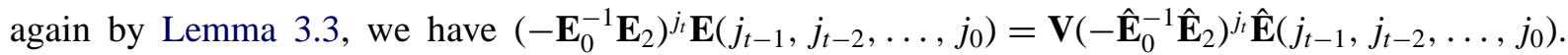
This means that Theorem 3.1 holds for $j_{t}$, i.e. $\mathbf{E}\left(j_{t}, j_{t-1}, j_{t-2}, \ldots, j_{0}\right)=\mathbf{V} \hat{\mathbf{E}}\left(j_{t}, j_{t-1}, j_{t-2}, \ldots, j_{0}\right)$, which complete the proof of Theorem 3.1.

Proof of Theorem 3.2. Theorem 3.2 is an immediate result of Theorem 3.1 by using Lemma 3.3 and the fact that $\mathbf{c}^{\mathrm{T}} \mathbf{V}=\hat{\mathbf{c}}^{\mathrm{T}}$.

\subsection{Comparison of the three methods}

All of the methods above have theory confirmation that the corresponding reduced model (8) can approximate the original model (3) by conserving some characteristics of the original transfer function or the unknown vector $\mathbf{x}$ in frequency domain.

As far as computational complexity is concerned, it is not so difficult to implement each of the methods. To implement Method 1, standard Arnoldi Process cannot be used, because elements $\mathbf{F}_{k}^{m}\left(\mathbf{E}_{0}^{-1} \mathbf{E}_{1}+\mathbf{E}_{0}^{-1} \mathbf{E}_{2}\right) \mathbf{E}_{0}^{-1} \mathbf{b}$ in (12) are not exactly the matrices in a Krylov subspace. It is mentioned by the end of [10] that $\mathbf{V}$ in (12) can be computed using a so-called Dual Arnoldi Algorithm which is a little more complicated than the standard Arnoldi Process.

For the second method, the columns in the projection matrix $\mathbf{V}$ in (15)are actually a group of orthogonal bases for the subspace that is composed of two Krylov subspaces, that is, $K_{J+1}^{s_{1}}=\left\{-\left(\mathbf{E}_{0}^{-1} \mathbf{E}_{1}\right), \mathbf{E}_{0}^{-1} \mathbf{b}\right\}$ and $K_{I+1}^{s_{2}}=\left\{-\left(\mathbf{E}_{0}^{-1} \mathbf{E}_{2}\right), \mathbf{E}_{0}^{-1} \mathbf{b}\right\}$. Standard Arnoldi Process can be employed to generate the orthogonal bases for these two Krylov subspaces, respectively [3,6], which makes realization of this method much easier.

The third method can also be implemented by standard Arnoldi Process or standard Block Arnoldi Process. For example, to generate spancol $\left\{\mathbf{V}_{0}\right\}=\operatorname{spancol}\left\{\bigcup_{j_{0}=0}^{q_{0}^{[0]}} \mathbf{E}\left(j_{0}\right)\right\}$, an orthogonal basis $\mathbf{V}_{0}$ of the Krylov subspace $K_{q_{0}^{[0]}+1}\left\{-\mathbf{E}_{0}^{-1} \mathbf{E}_{2}, \mathbf{E}_{0}^{-1} \mathbf{b}\right\}$ just needs to be generated.

Krylov subspace for the second projection matrix

$$
\operatorname{spancol}\left\{\mathbf{V}_{1}\right\}=\operatorname{spancol}\left\{\mathbf{E}\left(j_{0}\right), \mathbf{E}\left(j_{1}, j_{0}\right) j_{0}=0,1, \ldots, q_{0}^{[1]}, j_{1}=0,1, \ldots, q_{1}^{[1]}\right\}
$$

can be constructed based on $\mathbf{V}_{0}$ by using the relation $\mathbf{E}\left(j_{1}, j_{0}\right)=\left(-\mathbf{E}_{0}^{-1} \mathbf{E}_{2}\right)^{j_{1}} \mathbf{E}_{0}^{-1} \mathbf{E}_{1} \mathbf{E}\left(j_{0}\right)$. 
If we let $q_{0}^{[1]} \leq q_{0}^{[0]}$, then $\mathbf{V}_{1}$ can be computed by

$$
\operatorname{spancol}\left\{\mathbf{V}_{1}\right\}=K_{q_{1}^{[1]}+1}\left\{-\mathbf{E}_{0}^{-1} \mathbf{E}_{2}, \mathbf{B}_{1}\right\}, \quad \mathbf{B}_{1}=\mathbf{E}_{0}^{-1} \mathbf{E}_{1} \mathbf{V}_{0}\left(1: n_{1}\right) .
$$

Similarly, for $i \leq I, \mathbf{V}_{i}$ can be generated by

$$
\operatorname{spancol}\left\{\mathbf{V}_{i}\right\}=K_{q_{i}^{[i]}+1}\left\{-\mathbf{E}_{0}^{-1} \mathbf{E}_{2}, \mathbf{B}_{i}\right\}, \quad \mathbf{B}_{i}=\mathbf{E}_{0}^{-1} \mathbf{E}_{1} \mathbf{V}_{i-1}\left(1: n_{i}\right), \quad i=1, \ldots, I
$$

where $\mathbf{V}_{i-1}\left(1: n_{i}\right)$ means the first $n_{i}$ columns in $\mathbf{V}_{i-1}, i=1, \ldots, I$.

A common problem exists in the three methods is that there is not a prior error bound for the reduced model which is also the problem for conventional projection order reduction methods for one parameter system.

\subsection{Generalization to any parameter linear system}

Generalization of Method 1 in [10] to system (3) with any number $p$ parameters is studied in [11]. From (3), $\mathbf{x}$ can be expanded into series about $s_{1}, s_{2}, \ldots, s_{p}$ in the following way,

$$
\begin{aligned}
\mathbf{x} & =\left[\mathbf{I}-\left(s_{1} \mathbf{E}_{0}^{-1} \mathbf{E}_{1}+\cdots+s_{p} \mathbf{E}_{0}^{-1} \mathbf{E}_{p}\right)\right]^{-1} \mathbf{E}_{0}^{-1} \mathbf{b} u=\sum_{m=0}^{\infty}\left[\left(s_{1} \mathbf{E}_{0}^{-1} \mathbf{E}_{1}+\cdots+s_{p} \mathbf{E}_{0}^{-1} \mathbf{E}_{p}\right)\right]^{m} \mathbf{E}_{0}^{-1} \mathbf{b} u \\
& =\sum_{m=0}^{\infty} \sum_{k_{2}=0}^{m-\left(k_{3}+\cdots+k_{p}\right)} \ldots \sum_{k_{p-1}=0}^{m-k_{p}} \sum_{k_{p}=0}^{m}\left[\mathbf{F}_{k_{2}, \ldots, k_{p}}^{m}\left(\mathbf{E}_{0}^{-1} \mathbf{E}_{1}, \ldots, \mathbf{E}_{0}^{-1} \mathbf{E}_{p}\right) \mathbf{E}_{0}^{-1} \mathbf{b} u\right] s_{1}^{m-\left(k_{2}+\ldots+k_{p}\right)} s_{2}^{k_{2}} \ldots, s_{p}^{k_{p}}
\end{aligned}
$$

where $\mathbf{F}_{k_{2}, \ldots, k_{p}}^{m}$ is defined similarly as in (11). The projection matrix $\mathbf{V}$ is constructed as

$$
\begin{aligned}
\operatorname{spancol}\{\mathbf{V}\}= & \operatorname{spancol}\left\{\bigcup_{m=0}^{m_{q}} \bigcup_{k_{2}=0}^{m-\left(k_{p}+\cdots+k_{3}\right)} \ldots \bigcup_{k_{p-1}=0}^{m-k_{p}} \bigcup_{k_{p}=0}^{m} \mathbf{F}_{k_{2}, \ldots, k_{p}}^{m}\left(M_{1}, \ldots, M_{p}\right) \mathbf{b}_{M}\right\} \\
= & \operatorname{spancol}\left\{\mathbf{b}_{M}, M_{1} \mathbf{b}_{M}, M_{2} \mathbf{b}_{M}, \ldots, M_{p} \mathbf{b}_{M},\left(M_{1}\right)^{2} \mathbf{b}_{M},\left(M_{1} M_{2}+M_{2} M_{1}\right) \mathbf{b}_{M}, \ldots,\right. \\
& \left.\times\left(M_{1} M_{p}+M_{p} M_{1}\right) \mathbf{b}_{M},\left(M_{2}\right)^{2} \mathbf{b}_{M},\left(M_{2} M_{3}+M_{3} M_{2}\right) \mathbf{b}_{M}, \ldots\right\}
\end{aligned}
$$

where $M_{i}=-\mathbf{E}_{0}^{-1} \mathbf{E}_{i}, i=1,2, \ldots, p, \mathbf{b}_{M}=\mathbf{E}_{0}^{-1} \mathbf{b}$. And $\mathbf{F}_{k_{2}, \ldots, k_{p}}^{m}\left(M_{1}, \ldots, M_{p}\right)$ can be generated recursively as

$$
\begin{aligned}
& \mathbf{F}_{k_{2}, \ldots, k_{p}}^{m}\left(M_{1}, \ldots, M_{p}\right) \\
& = \begin{cases}0, & \text { if } k_{i} \nsubseteq\{0,1, \ldots, m\}, i=2, \ldots, p \\
0, & \text { if } k_{2}+\cdots+k_{p} \nsubseteq\{0,1, \ldots, m\} \\
\mathbf{I}, & \text { if } m=0 \\
M_{1} \mathbf{F}_{k_{2}, \ldots, k_{p}}^{m-1}\left(M_{1}, \ldots, M_{p}\right)+M_{2} \mathbf{F}_{k_{2}-1, \ldots, k_{p}}^{m-1}\left(M_{1}, \ldots, M_{p}\right)+\ldots+M_{p} \mathbf{F}_{k_{2}, \ldots, k_{p}-1}^{m-1}\left(M_{1}, \ldots, M_{p}\right)\end{cases}
\end{aligned}
$$


It is easy to see that for system with more than two parameters, method in [11] will be more complicated. In the experimental examples in [11], only the first few $\mathbf{F}$ with $m=0,1$ were computed to generate the projection matrix $\mathbf{V}$ and the reduced models of the examples shown there are already accurate enough. However, for some other very large sized systems (for example, those from finite element discretization) higher order $\mathbf{F}(m>1)$ probably need to be computed to increase the accuracy of the reduced model. In this case, it is difficult to use the Arnoldi Process or the Dual Arnoldi Algorithm to implicitly generate $\mathbf{F}_{k_{2}, \ldots, k_{p}}^{m-1}\left(M_{1}, \ldots, M_{p}\right)$. If $\mathbf{F}_{k_{2}, \ldots, k_{p}}^{m-1}\left(M_{1}, \ldots, M_{p}\right)$ are first computed explicitly and then orthogonalized to form the projection matrix $\mathbf{V}$, high numerical instability will be caused, which is similar as that in [1] whose numerical instability was analyzed and improved in [2] by using Krylov subspace to implicitly compute the moments of the transfer function for the one parameter system (1).

Generalization of Method 2 to more parameters system (3) is natural [13], just compute all the derivatives of $\mathbf{x}\left(s_{1}, \ldots, s_{p}\right)$ at $s_{i}=0, i=1,2, \ldots, p$, respectively, by assuming that other parameters $s_{j}, j \neq i$ are constant numbers $s_{j}^{0}$, that is, define $\tilde{\mathbf{E}}_{0}=\mathbf{E}_{0}+\sum_{j \neq i} s_{j}^{0} \mathbf{E}_{j}$, denote $M_{k}^{s_{i}}$ the $k$ th order derivative of $\mathbf{x}$ at $s_{i}=0, i=1, \ldots, p$,

$$
\left(\tilde{\mathbf{E}}_{0}+s_{i} \mathbf{E}_{i}\right)\left[M_{0}^{s_{i}}+M_{1}^{s_{i}} s_{i}+\cdots \frac{M_{k}^{s_{i}}}{k !}\left(s_{i}\right)^{k}+\cdots\right]=\mathbf{b} u
$$

Like powers of $s_{i}$ being equated, the moments can be computed recursively,

$$
\begin{aligned}
& \tilde{\mathbf{E}}_{0} M_{0}^{s_{i}}=\mathbf{b} u \\
& \tilde{\mathbf{E}}_{0} M_{k}^{s_{i}}=-k \mathbf{E}_{i} M_{k-1}^{s_{i}}, \quad k=1,2, \ldots
\end{aligned}
$$

The projection matrix $\mathbf{V}$ is just derived by orthogonalization of certain number of derivatives of $\mathbf{x}$ at every parameter,

$$
\operatorname{spancol}\{\mathbf{V}\}=\operatorname{spancol}\left\{M_{0}^{s_{1}}, M_{1}^{s_{1}}, \ldots, M_{q_{1}}^{s_{1}}, M_{0}^{s_{2}}, M_{1}^{s_{2}}, \ldots, M_{q_{2}}^{s_{2}}, \ldots, M_{0}^{s_{p}}, M_{1}^{s_{p}}, \ldots, M_{q_{p}}^{s_{p}}\right\}
$$

From (26), it is clear that the projection matrix can be easily generated by performing several times of Arnoldi Process.

For Method 3, it can certainly deal with more parameters, just doing further more expansions about other parameters $s_{i}, i>2$ successively. The expression will become longsome, but the standard Block Arnoldi Process can still be employed to implicitly generate the projection matrices.

Being compared with two parameter model reduction, the reduction process of any of the methods above for more parameter system are much more complicated. For example, there are much more numbers have to be decided before computing the projection matrix $\mathbf{V}$, such as $m, k_{2}, \ldots, k_{p}$ in $\mathbf{F}(24) ; q_{1}, q_{2}, \ldots, q_{p}$ in (27); $j_{i}, i=1, \ldots, I$ in $\mathbf{E}$ (19). How to choose each number properly is uncertain. By using large enough numbers can usually get accurate reduced model, but the number of the columns in the projection matrix $\mathbf{V}$ will thereby increase fast, which means that a smaller sized reduced model can not be obtained by projection $\mathbf{x} \approx \mathbf{V z}$. Therefore, at present, choice of the number of matrices $\mathbf{F}_{k_{1}, \ldots, k_{p}}^{m} ; M_{i}^{s_{j}} ; \mathbf{E}\left(j_{i}, j_{i-1}, \ldots, j_{0}\right)$ included in the projection matrix is just empirical, which implicit that one-time order reduction may not get a satisfactory reduced model. The second-time or even the third-time model order reduction have to be done by changing the number of derivatives included in the projection matrix. 


\section{Model order reduction on nonlinear parameter system}

\subsection{Overview}

The nonlinear parameter system is defined as

$$
\begin{aligned}
& \mathbf{H}\left(s_{1}, \ldots, s_{p}\right) \mathbf{x}\left(s_{1}, \ldots, s_{p}\right)=\mathbf{b} u \\
& y=\mathbf{c}^{\mathrm{T}} \mathbf{x}
\end{aligned}
$$

where $\mathbf{H}$ is nonlinearly dependent on the parameters $s_{i}, i=1, \ldots, p$.

Method in [11] first approximate (28) by a system as in (3) by power-series expansion of $\mathbf{H}$ on $s_{i}, i=1, \ldots, p$, then order reduction is done on (3) instead, which can be solved by the method in Section 3. One easy way to expand $\mathbf{H}$ into series of $s_{i}, i=1, \ldots, p$, is using Taylor series expansion.

$$
\mathbf{H}\left(s_{1}, \ldots, s_{p}\right)=M_{0}+\sum_{i} M_{i}+\sum_{h, k} s_{h} s_{k} M_{h, k}+\sum_{h, k, j} s_{h} s_{k} s_{j} M_{h, k, j}+\cdots
$$

By defining a new group of matrices and parameters

$$
\begin{aligned}
& \tilde{\mathbf{E}}_{i}= \begin{cases}M_{i}, & i=0, \ldots, \mu \\
M_{h, k}, & h=1, \ldots, \mu ; k=1, \ldots, \mu \\
M_{h, k, j}, & h=1, \ldots, \mu ; k=1, \ldots, \mu ; j=1, \ldots, \mu\end{cases} \\
& \tilde{s}_{i}= \begin{cases}s_{i}, & i=0, \ldots, \mu \\
s_{h} s_{k}, & h=1, \ldots, \mu ; k=1, \ldots, \mu \\
s_{h} s_{k} s_{j}, & h=1, \ldots, \mu ; k=1, \ldots, \mu ; j=1, \ldots, \mu\end{cases}
\end{aligned}
$$

system (28) is approximated by a linear parameter system by truncating the higher order terms in Taylor series (29).

$$
\begin{aligned}
& \left(E_{0}+\tilde{s}_{1} \tilde{\mathbf{E}}_{1}+\tilde{s}_{2} \tilde{\mathbf{E}}_{2}+\cdots+\tilde{s}_{p} \tilde{\mathbf{E}}_{p}\right) \mathbf{x}=\tilde{\mathbf{b}} u \\
& y=\mathbf{c}^{\mathrm{T}} \mathbf{x}
\end{aligned}
$$

The form of this linear parameter system (32) is no different from that of (3) therefore, (22)-(24)in Section 3 can be employed to do model order reduction.

The other method in [12,13] directly deals with system (28) by computing derivatives of both $\mathbf{H}$ and $\mathbf{x}$ at parameters $s_{i}, \ldots, s_{p}$, respectively, while keeping the other parameters as constant numbers, i.e. $s_{j}=s_{j}^{0}, j \neq i$. For example, denote $H_{k}^{s_{i}}$ the $k$ th order derivative of $\mathbf{H}$ w.r.t. the $i$ th parameter $s_{i}$ at $s_{i}=0$ and $M_{k}^{s_{i}}$ the $k$ th order derivative of $\mathbf{x}$ at $s_{i}=0$, then,

$$
\left(H_{0}^{s_{i}}+s_{i} H_{1}^{s_{i}}+\cdots+\frac{1}{k !}\left(s_{i}\right)^{k} H_{k}^{s_{i}}+\cdots\right)\left(M_{0}^{s_{i}}+s_{i} M_{1}^{s_{i}}+\cdots+\frac{1}{k !}\left(s_{i}\right)^{k} M_{k}^{s_{i}}+\cdots\right)=\mathbf{b} u
$$

$M_{k}^{s_{i}}$ can thus be computed by equating like powers of $s_{i}$ on both sides of (33),

$$
\begin{aligned}
& H_{0}^{s_{i}} M_{0}^{s_{i}}=\mathbf{b} u \\
& H_{0}^{s_{i}} M_{k}^{s_{i}}=\left(-\sum_{r=1}^{k} \frac{H_{r}^{s_{i}} M_{k-r}^{s_{i}}}{r !(k-r) !}\right) k !, \quad k=1, \ldots
\end{aligned}
$$


These derivatives can be computed recursively, but can never be computed implicitly by Arnoldi Process by which good numerical stability can be conserved. How to compute these derivatives efficiently was not discussed in [13], but it is definitely not as easy as the linear case.

\subsection{Approach to avoid numerical instability}

A better way to avoid possible numerical instability exists in both [11] and [12,13] for nonlinear parameter system is to synthesize the two methods together by dropping their weakness.

Since method in $[12,13]$ can easily deal with linear parameter systems with standard Arnoldi Process being employed to implicitly generate the projection matrix $\mathbf{V}$, we can first approximate the nonlinear parameter system (28) by a linear parameter system with (29)-(32) and then compute the projection matrix using (25)-(27) so that numerical stability can be maintained well with the computational complexity being reduced too.

\section{Numerical experiments}

In this section, we illustrate the robustness of parametric model order reduction technique by a simple example, which comes from a thermal problem, a microthruster unit. More detail about the related device is given in [14]. The parameter model constructed from this problem is the system as follows,

$$
\begin{aligned}
& \mathbf{C} \frac{\mathrm{d} \mathbf{x}(t)}{\mathrm{d} t}+(\mathbf{G}+h \mathbf{D}) \mathbf{x}(t)=\mathbf{B} \\
& y(t)=\mathbf{E x}(t)
\end{aligned}
$$

where $h$ is a parameter, it is usually called the "film coefficient". The change range of $h$ can be from 1 to $10^{9}$. The dimension of this system is $n=4257$. The output $y(t)$ represents the temperature in the center of the heater in the device.

What is interested in engineering applications is how the temperature $y(t)$ changes with $h$. With the traditional simulation tool, in order to observe the output $y(t)$ with different values of $h$, we have to simulate the whole system whenever $h$ is set to a new value. If we need to observe $y(t)$ corresponds to thousands of values of $h$, then we have to simulate this system for thousands of times, which will be very time consuming. In the following, we will obtain a reduced small model by parametric model reduction to approximate the original large model, then we only need to simulate this small model every time $h$ changes. To apply the parametric model reduction, we first do Laplace transformation on (35)and get,

$$
\begin{aligned}
& s \mathbf{C X}+(\mathbf{G}+h \mathbf{D}) \mathbf{X}=\mathbf{B} \\
& y(t)=\mathbf{E X}
\end{aligned}
$$

from (36), it is just the case in (3) when $p=2$; therefore, we can construct a projection matrix $\mathbf{V}$ by any one of the method introduced in Section 3. Here, we employ the Method 3 in Section 3.3 to show the efficiency of our proposed method.

The projection matrix $\mathbf{V}$ is constructed as follows,

$$
\operatorname{spancol}\{\mathbf{V}\}=\operatorname{spancol}\left\{\mathbf{V}_{0}, \mathbf{V}_{1}, \mathbf{V}_{2}\right\}
$$




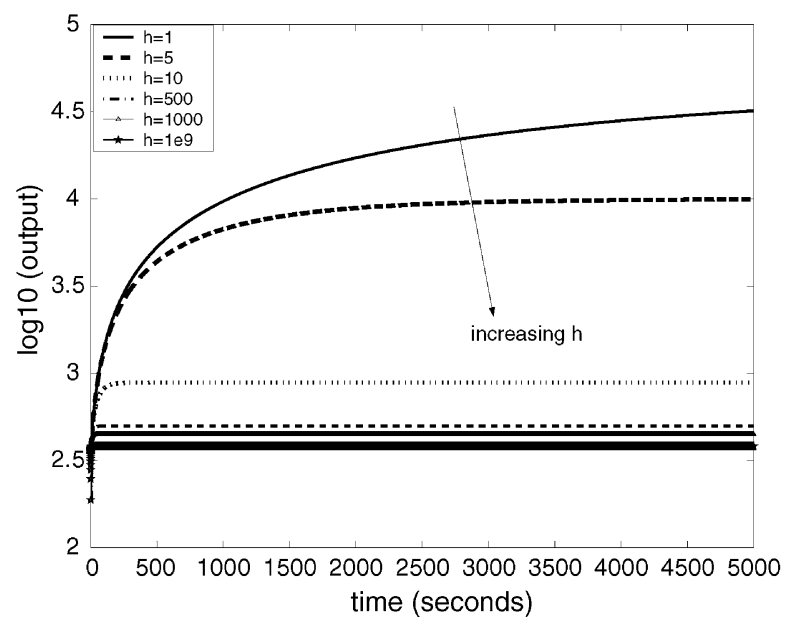

Fig. 1. Outputs with different values of $h$.

where

$$
\operatorname{spancol}\left\{\mathbf{V}_{0}\right\}=\operatorname{spancol}\left\{\mathbf{G}^{-1} \mathbf{B}, \mathbf{G}^{-1} \mathbf{D} \mathbf{G}^{-1} \mathbf{B}, \ldots,\left(\mathbf{G}^{-1} \mathbf{D}\right)^{q_{0}^{[0]}} \mathbf{G}^{-1} \mathbf{B}\right\}
$$

Denote $\mathbf{B}_{1}=\mathbf{C V}_{0}\left(1: j_{0}\right), j_{0} \leq q_{0}^{[0]}+1$,

$$
\operatorname{spancol}\left\{\mathbf{V}_{1}\right\}=\operatorname{spancol}\left\{\mathbf{G}^{-1} \mathbf{B}_{1}, \mathbf{G}^{-1} \mathbf{D} \mathbf{G}^{-1} \mathbf{B}_{1}, \ldots,\left(\mathbf{G}^{-1} \mathbf{D}\right)^{q_{1}^{[1]}} \mathbf{G}^{-1} \mathbf{B}_{1}\right\}
$$

Denote $\mathbf{B}_{2}=\mathbf{C V}_{1}\left(1: j_{1}\right), j_{1} \leq q_{1}^{[1]}+1$,

$$
\operatorname{spancol}\left\{\mathbf{V}_{2}\right\}=\operatorname{spancol}\left\{\mathbf{G}^{-1} \mathbf{B}_{2}, \mathbf{G}^{-1} \mathbf{D} \mathbf{G}^{-1} \mathbf{B}_{2}, \ldots,\left(\mathbf{G}^{-1} \mathbf{D}\right)^{q_{2}^{[2]}} \mathbf{G}^{-1} \mathbf{B}_{2}\right\}
$$

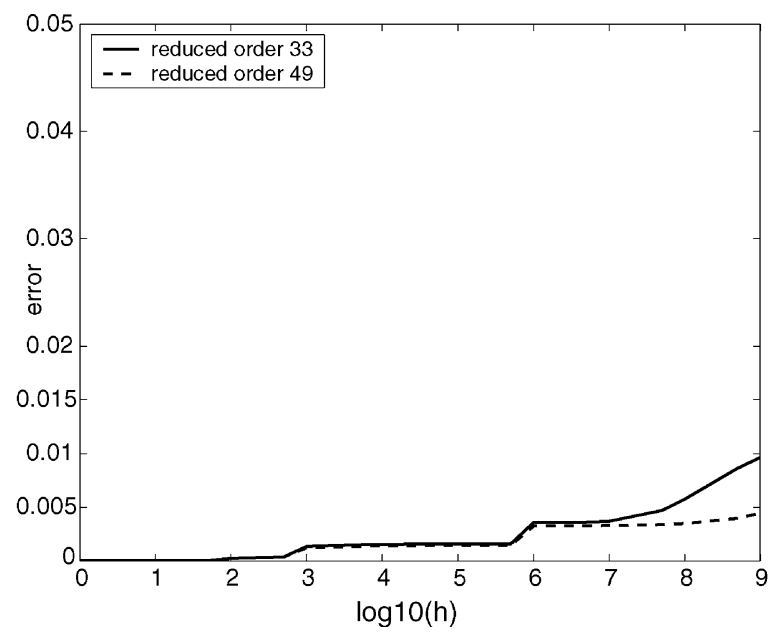

Fig. 2. Error of parametric model order reduction. 
From the construction above, it is easy to see that the three matrices $\mathbf{V}_{0}, \mathbf{V}_{1}, \mathbf{V}_{2}$ can be generated by standard Arnoldi Process. With this projection matrix $\mathbf{V}$, we obtain a reduced small system by approximate the original solution vector $\mathbf{x}$ with $\mathbf{x} \approx \mathbf{V z}$, them multiply the transpose of $\mathbf{V}$ on both sides of the derived equation, we have,

$$
\begin{aligned}
& \mathbf{V}^{\mathrm{T}} \mathbf{C V} \frac{\mathrm{d} \mathbf{z}(t)}{\mathrm{d} t}+\mathbf{V}^{\mathrm{T}}(\mathbf{G}+h \mathbf{D}) \mathbf{V z}(t)=\mathbf{V}^{\mathrm{T}} \mathbf{B} \\
& y(t)=\mathbf{E V z}(t)
\end{aligned}
$$

the final reduced small system in the time domain is

$$
\begin{aligned}
& \hat{\mathbf{C}} \frac{\mathbf{z}(t)}{\mathrm{d} t}+(\hat{\mathbf{G}}+h \hat{\mathbf{D}}) \mathbf{z}(t)=\hat{\mathbf{B}} \\
& y(t)=\hat{\mathbf{E}} \mathbf{z}(t)
\end{aligned}
$$

where $\hat{\mathbf{C}}=\mathbf{V}^{\mathrm{T}} \mathbf{C V}, \hat{\mathbf{G}}=\mathbf{V}^{\mathrm{T}} \mathbf{G V}, \hat{\mathbf{D}}=\mathbf{V}^{\mathrm{T}} \mathbf{D V}, \hat{\mathbf{B}}=\mathbf{V}^{\mathrm{T}} \mathbf{B}, \hat{\mathbf{E}}=\mathbf{E V}$.

We show in Fig. 1 the outputs of this system corresponding to different values of $h$. We use the logarithm scale in the figure in order to put all the considered outputs into one figure. It can be seen that the magnitude of the output will change rapidly once the value of $h$ is changed.

In Fig. 2, we give the results of parametric model order reduction, the errors between $\hat{y}(t)$ in (38) and $y(t)$ in (35) corresponding to different values of $h$ are plotted. The error corresponding to a fixed $h$ is defined as error $=\|\hat{y}(t)-y(t)\| /\|y(t)\|$. The solid line is the error plot of the reduced system (38) whose dimension is $q=33$. All the errors are below 0.01 , which is already accurate enough for engineering applications $[15,16]$. If we want a more accurate small model, we can increase the dimension of the reduced system (38), the dotted line is the error plot of the reduced system (38), whose dimension is $q=49$. The errors are all below 0.005 . Notice that the values of $h$ change from 0 to $10^{9}$, which is a very large range. Therefore, we conclude that the reduced system (38) can keep acceptable accuracy while allow the parameter $h$ changes widely, it is a parameter independent reduced model.

\section{Conclusions}

We analyzed several recently developed model order reduction methods for parameter systems. The basic idea is by projecting the original full unknown vector into a lower dimension subspace to get a reduced small dimensional model. The goal is how to construct a projection matrix so that the resulting reduced model can approximate the original large full model as accurate as possible for all the parameters and the computation complexity is also acceptable. For a system with two or three parameters all these methods are relatively easy to implement, while for the system with more parameters they will become more complicated. An alternative method is introduced for linear parameter system and an improved method for nonlinear parameter system model order reduction is given. Numerical experiments also show the efficiency of the parametric model order reduction technique and confirm the theoretical analysis. As far as all these methods are concerned, there is not a prior computable error bound for the reduced model, which is the drawback of model order reduction methods with projection technique based on Krylov subspace. More work should be done in this direction and other more complicated engineering examples with more than two parameters for an experimental comparison of accuracy and applicability of these methods will be given in the future work. 


\section{Acknowledgements}

This research is supported partly by NSFC research project 90307017, 60176017, 90207002, Synopsys Inc., Cross-Century Outstanding Scholar's fund of Ministry of Education of China, National 863 Plan Projects 2002AA1Z1340 and 2002AA1Z1460, the Doctoral Program Foundation of Ministry of Education of China 2000024628, Science \& Technology Key Project of Ministry of Education of China 02095, Shanghai Science and Technology Committee Project 01JC14014, Shanghai AM R\&D fund 0107, NSF grants CCR-0098275 and CCR-0306298 and fund of Alfried Krupp von Bohlen und Halbach-Stiftung of Germany.

\section{References}

[1] L.T. Pillage, R.A. Rohrer, Asumptotic waveform evaluation for timing analysis, IEEE Trans. Comput. Aided Des. 9 (April 1990) 325-366.

[2] P. Feldmann, R.W. Freund, Efficient linear circuit analysis by Padé approximation via the Lanczos process, IEEE Trans. CAD 14 (5) (May 1995).

[3] L. Miguel Silveira, M. Kamon, J.K. White, Efficient reduced-order modeling of frequency-dependent coupling inductances associated with 3-d interconnect structures, in: Proceedings of the 32nd Design Automation Conference, June 1995, pp. 376-380.

[4] P. Feldmann, R.W. Freund, Reduced-order modeling of large linear subcircuits via a block Lanczos algorithm, in: IEEE/ACM Proceedings of DAC, June 1995, pp. 474-479.

[5] E.G. Grimme, Krylov projection methods for model reduction, Ph.D. Dissertation, University of Illinois, UrbanaChampaign, 1997.

[6] R. Freund, Reduced order modeling techniques based on Krylov subspaces and their use in circuit simulation, in: Numerical Analysis Manuscript 98-3-02, Bell Laboratory, Murray Hill, NJ, February 1998.

[7] L.M. Silvera, M. Kamon, I. Elfadel, J. White, A coordinate transformed Arnoldi algorithm for generating guaranteed stable reduced order models arbitrary RLC circuits, in: In IEEE/ACM Proceedings of ICCAD, November 1996, pp. $288-294$.

[8] Zhaojun Bai, P.M. Dewilde, R.W. Freund, Reduced order modeling, in: Numerical Analysis Manuscript No. 02-4-13, Bell Laboratory, Murray Hill, NJ, March 2002, available on line at http://cm.bell-labs.com/cs/doc/02.

[9] J. Roychowdhury, Reduced-order modelling of linear time-varying systems, in: Proceedings of IEEE ICCAD, 1998, pp. 92-95.

[10] D.S. Weile, E. Michielssen, E. Grimme, K. Gallivan, A method for generating rational interpolant reduced order models of two-parameter linear systems, Appl. Math. Lett. 12 (1999) 93-102.

[11] L. Daniel, O.C. Siong, L.S. Chay, K.H. Lee, J. White, A multiparameter moment-matching model-reduction approach for generating geometrically parameterized interconnect performance models, IEEE Trans. Comput. Aided Des. Integr. Circuits Syst. 23 (5) (May 2004).

[12] P. Gunupudi, M. Nakhla, Multi-dimensional model reduction of VLSI interconnects, in: Proceedings of IEEE Custom Intergrated Circuits Conference, May 2000, pp. 499-502.

[13] P. Gunupudi, R. Khazaka, M. Nakhla, T. Smy, D. Celo, Passive parameterized time-domain macromodels for high-speed transmission-line networks, IEEE Trans. Microwave Theory Tech. 51 (December 12) (2003).

[14] T. Bechtold, E.B. Rudnyi, J.G. Korvink, Automatic generation of compact electro-thermal models for semiconductor devices, IEICE Trans. Electron. E86C (2003) 459-465.

[15] C.J.M. Lasance, Recent progress in compact thermal models, in: 19th IEEE SEMI-THERM Symposium, 2003.

[16] C.J.M. Lasance, Two benchmarks to facilitate the study of compact thermal modeling phenomena, IEEE Trans. Components Packaging Technol. 24 (2001) 559-565. 